\title{
Neon color spreading and structural information measures
}

\author{
H. F. J. M. van TUIJL and E. L. J. LEEUWENBERG \\ University of Nijmegen, Erasmuslaan 16, Nijmegen, The Netherlands
}

\begin{abstract}
This article presents a study on Van Tuijl's (1975) neon effect. The neon effect can be described as an illusory spreading of color around the colored elements of an otherwise black line pattern. The observer has a strong impression of colored light projected onto a lattice of black lines. The hypothesis is advanced that the neon effect will only result if the structural relationships between black and colored line elements in the pattern are such that a neon interpretation is the most efficient interpretation that can be given of the pattern. The necessity of this approach to the neon phenomenon emanates from the inadequacy of alternative, more simple, explanations, such as aberrations of peripheral perceptual mechanisms or the presence in the pattern of easily definable stimulus features. To subject the hypothesis proposed above to experimental test, a precise quantification of its central concept, the efficiency of pattern interpretations, is needed. To that end, Leeuwenberg's (1971) coding language for sequential patterns is introduced. By means of the coding language, pattern interpretations can be represented in a pattern code, the length of which is inversely proportional to the efficiency of the interpretation coded. Several possible interpretations of color differences between the elements of line patterns are discussed, and it is shown how the efficiency of each of them can be determined. Next, in two experiments, the efficiency of the neon interpretation relative to that of alternative interpretations of color differences in line patterns is varied, by manipulating the structural relations between black and colored line elements, and the dependency of the neon effect on the relative efficiency of the neon interpretation is demonstrated. Implications of the findings are discussed.
\end{abstract}

Recently, a remarkable color-spreading phenomenon, called neon effect, was discovered (Van Tuijl, 1975). The effect may occur if, in a lattice of uniformly colored lines, some lines are given a different color (see Figure 1a for an illustration of the effect). ${ }^{1}$ Instead of seeing a lattice with a particular subpattern standing out, because of its deviating color, one has the impression that the lattice is illuminated by a source shedding colored light on the area in which the deviant lines lie. So, in the example of Figure 1a, the color seems to transgress the limits of the actual colored lines and to spread out over an area that is in reality uncolored. Moreover, it is at first sight practically impossible to give an accurate description of the structure made up by the colored line elements. They are totally embedded within the whole of the lattice, and, in addition, they seem to be more vague than the black lines. It is, in particular, the embeddedness and vagueness of the colored lines, together with the impression of the pattern as a unitary lattice functioning as a background for the

Requests for reprints should be sent to H. F. J. M. van Tuijl, Department of Psychology, University of Nijmegen, Erasmuslaan 16, Nijmegen, The Netherlands. The first author's share in the preparation of this article was made possible by a grant from the Netherlands Organization for the Advancement of Pure Research (Z.W.O.). The authors wish to thank Ch. M. M. de Weert and F. Restle for their critical comments on a first draft. light projected onto it, which form the defining features of the neon effect, compared with other spreading phenomena already known. ${ }^{2}$

At this moment, we are not concerned with the phenomenon of spreading, as such, but with the specific quality of neon spreading and the conditions under which this specific kind of spreading turns up. Our position is that, if some preconditions discussed elsewhere (Van Tuijl \& De Weert, Note 1) are met, the neon effect will arise in a pattern if a neon interpretation is the most efficient interpretation that can be given of the pattern. By neon interpretation, we mean an interpretation according to which color differences in a line pattern are caused by an independent source projecting colored light onto a differently colored lattice. By saying that the perceiver chooses the most efficient interpretation that fits the stimulus, we take the Gestalt law of Prägnanz (Koffka, 1935, p. 110) as our point of departure. This approach was suggested in an earlier article (Van Tuijl, 1975), in which the striking difference in neon effect between the left and right pattern in Figure 1b was tentatively explained by the difference in relative efficiency of the neon interpretation in these patterns. In the case of the pattern at the left, the neon interpretation was said to be the most efficient one, whereas the right pattern could better be described as three steplike structures, one of which was colored in a deviating way. In this article, we want 


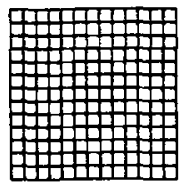

$1 a$

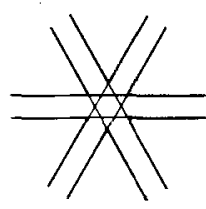

1d

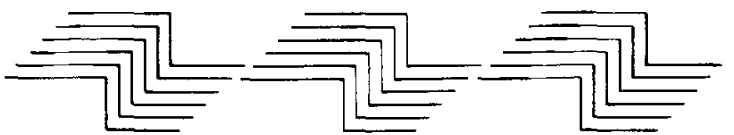

(a)

$1 \mathrm{~g}$

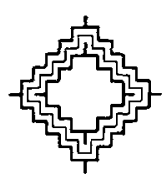

(b) (b)

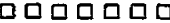

व $\square \square \square \square$ ㅁㅁㅁ 믐ㅁㅁㅁำ

$1 \mathrm{c}$

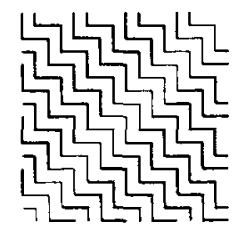

le

(c)

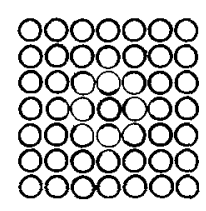

$1 f$
ㅁㅁㅁ

ロ ロ •ロ

Figure 1. (a) A typical example of a neon displaying pattern. (In this and all further illustrations, thin lines are used instead of colored ones.) The entire area in which the deviant lines lie seems to be illuminated by a colored light source. (b) Two identical colored step structures, surrounded in different ways by identical black (sub)structures, resulting either in a substantial neon effect (a) or in no neon effect at all (b). (c) A neon effect occurs, although black and colored line elements are not interconnected. (d) No neon effect occurs, although black and colored line elements are both connected and of identical orientation. (e) $A$ neon effect occurs, although black and colored line elements are of different orientations. (f) $A$ neon effect occurs, although the pattern is made up of curved rather than straight lines. (g) The neon interpretation of all three patterns is identical and therefore equally efficient in all three cases. The patterns do differ with regard to the efficiency of an alternative, nonneon, interpretation, which can be given to them. Differences in neon effect between a, $b$, and $c$ are supposed to result from the different relative efficiencies of their neon interpretations.

to scrutinize the hypothesis that the neon effect depends on the relative efficiency of the neon interpretation, by using an objective measure for the efficiency of pattern interpretations, so that unambiguous, quantitative predictions can be made. To that end, we will introduce Leeuwenberg's coding language for the representation of visual pattern interpretations (Leeuwenberg, 1971), by means of which the amount of structural information of a pattern interpretation can be determined. The less the amount of structural information of an interpretation, the more efficient it is. Secondly, we want to determine if not only the occurrence of the neon effect, but also the strength with which it occurs can be related to the relative efficiency of the neon interpretation.

First some demonstrations that argue against less cognitive explanations for the specific quality of neon spreading are presented. Next, the Leeuwenberg coding language will be introduced as far as necessary for the understanding of its application to the phenomenon under investigation. After that, two experiments will be discussed in which the dependency of the neon effect on the relative efficiency of the neon interpretation is examined.

\section{POSSIBLE EXPLANATIONS FOR THE NEON EFFECT}

Figure $1 \mathrm{~b}$ presents two patterns; in the left one, a clear neon effect is shown, whereas in the right one, practically no neon can be seen at all. Without providing full proof of the explanation advocated by us, at least some alternative explanations are discredited by this demonstration.

First, the neon effect cannot be the result of aberrations of peripheral perceptual mechanisms, such as, e.g., malfunction of specific color receptors, because, if such were the case, there would be no reason why the neon effect would not occur in both patterns of Figure 1b. Chromatic aberration cannot be a tenable explanation, either. Chromatic aberration refers to the fact that the lens of the eye, being not entirely achromatic, cannot produce sharp images of lines of all wavelengths at the same time. Therefore, the neon effect could be the result of imprecise representation of colored lines while the eye focuses on black ones. However, we cannot think of any reason why such an effect would not be equally strong in both patterns of Figure $1 \mathrm{~b}$.

Second, the factors which Helson (1963) showed to affect the occurrence of line spreading in the Von Bezold pattern, such as line width, line separation, line reflectance, and background reflectance, are not sufficient to explain the occurrence of the neon effect. Narrow, dark lines at short distances on a high-reflectance background ought to spread, according to Helson. However, the right pattern in Figure $1 \mathrm{~b}$ does not show a neon effect, as does the left one, and moreover, it hardly shows any spreading at all. So it would be worthwhile to extend the research on the Von Bezold effect to structural factors, too.

Also, Beck's (1966) observation that lines darker than the background spread as opposed to lines which are lighter than the background cannot represent a general rule, as is illustrated by the right pattern in Figure 1b. Moreover, it has been shown (van Tuijl, 1975 ) that the neon effect can occur if light lines are placed on a dark background.

Before claiming the necessity of a structural approach to our phenomenon, we will look for other pattern features, on the basis of which patterns that show a neon effect can be discriminated from patterns that do not show such an effect. In examining the patterns in Figure 1b, some candidates can be easily 
found. A striking difference between the two is the connectedness of black and colored elements in the left one and the absence of such connections in the right one. In addition, in the left pattern, all colored elements have the same orientation as the black elements with which they are connected. Therefore, black and colored line elements could be analyzed by the same line detectors, and the neon effect could be the result of an incongruity caused by simultaneous stimulation of the same line detectors but different color receptors.

With regard to a possible relationship between neon effect and connectedness of black and colored line elements, two points are relevant. Connectedness could be a necessary condition for the occurrence of neon, and, as well, could be a sufficient condition. To exclude the former, we have to demonstrate the occurrence of neon effect in a pattern in which black and colored elements are not connected. This situation is realized in the pattern in Figure 1c. To exclude the latter, a pattern has to be designed in which black and colored elements are connected but in which no neon shows up. Indeed, no neon effect is manifest in the pattern illustrated in Figure 1d. At the same time, this pattern disproves part of the suggestion that the second pattern aspect mentioned above, viz., similarity of the orientation of black and colored elements could explain the occurrence of the neon effect. Notwithstanding the fact that black and colored elements have been drawn in the same orientation, no neon effect occurs. So, identical orientations appear to be no sufficient condition to evoke a neon effect. Identity of orientation is not even a necessary condition, as can be seen in Figure 1e, in which a neon appears even though black and colored elements do not have identical orientations.

Apart from the two pattern aspects mentioned, which have been shown not to be critical for the occurrence of the neon effect, a number of aspects pertaining to both patterns in Figure 1b can be enumerated, which may be necessary, though apparently not sufficient, conditions for the occurrence of neon effect. It might be that, in order to show neon, patterns must be characterized by one or more of the following features, which have to be present, independent of the efficiency of the neon interpretation: (a) straight lines rather than curved ones, (b) a regularly structured pattern rather than a random one, (c) regular contours of the subjectively colored neon area, (d) regularity of the structure of the colored line elements, and (e) a large number of black and colored line elements. All these aspects have been extensively studied, however, without a positive result. It can be demonstrated very easily (see Figure 1f) that neon can occur in patterns made up of curved rather than straight lines. The effect cannot, therefore, be dependent on specific line-detecting mechanisms.
Furthermore, the occurrence of neon can be demonstrated in random patterns, and neither the contours of the subjectively colored area nor the structure of the group of actually colored line elements have to be regular in order to evoke a neon. The reader can easily convince himself by drawing an irregular set of black lines and replacing parts of those lines by colored line elements in an unsystematic way. Regarding the last point mentioned above, it can be stated that neon can be evoked with only a very few lines. So, although a lower limit has not actually been established, there seems to be no need to require a large number of lines or a texture in order for the effect to show up.

We will now discuss in greater detail our position examining some patterns, of which the relative efficiency of the neon interpretation can be estimated without going into the details of the Leeuwenberg coding language. Looking at the patterns in Figure $1 \mathrm{~g}$, it is clear that their overall structure is identical. All three can be interpreted as a set of parallel lines, which change their direction two times. One could add to the description of each pattern that the color in it is caused by the projection of an oblong, colored figure. In other words, the neon interpretation of all three patterns is identical and so has to be equally efficient in all three cases. However, the patterns happen to differ with regard to the magnitude of the neon effect shown by them. As we have seen above (see Figure 1e), these differences cannot be explained by the changes in direction that accompany the color changes in Patterns $b$ and $c$ in Figure 1g. The reason for their occurrence is, in our opinion, to be looked for in the circumstance that the neon interpretation, only in the case of Patterns a and $c$, is the most efficient interpretation that can be given of the pattern. In the case of Pattern $b$, the going together of form and color changes allows for an alternative interpretation that is equally as efficient as the neon interpretation of the pattern. According to this alternative interpretation, the pattern is conceived of as a lattice of differently colored line elements. Such an interpretation is highly efficient, because the positions where the color changes take place are identical to the positions where the form changes take place, and, therefore, the former can be related to the latter. In Pattern a, however, form and color changes do not occur together. Therefore, an alternative interpretation, such as the one given for Pattern $b$, would, in the case of Pattern a, be rather inefficient, because the places in the pattern where the color transitions take place would have to be specially indicated.

The reader might wonder why the same argument does not hold for the pattern in Figure 1e, which combines a neon effect with parallel form and color changes. The critical difference between that pattern 
and Pattern $b$ in Figure 1g, however, is that, in the latter, all form changes are accompanied by a change of color, whereas in the case of the pattern in Figure 1e, only a certain number of form changes have color changes in parallel. The nonneon interpretation would, in that case, become comparatively inefficient, because it would have to specify precisely which form changes are accompanied by color changes. Pattern $\mathrm{c}$ in Figure $1 \mathrm{~g}$ is intermediate between Patterns $a$ and $b$ with regard to the relative efficiency of the neon interpretation: One change of color in the pattern is coupled to a form change, whereas the other one is not. Therefore, the first color transition can be just as efficiently indicated by means of a neon figure superimposed on the pattern as by adding a color change to each line's change of direction, which had to be indicated anyway. The second color transition, however, would require an extra indication for its position, as well as an indication for the color change itself, which makes the alternative interpretation a little less efficient compared with the neon interpretation. In any case, the alternative, nonneon interpretation of Pattern $c$ is certainly more complex than the alternative interpretation of Pattern $b$, but less complex than that of Pattern a, whereas the neon interpretations of all three patterns are equally efficient. At the same time, it can be seen that the magnitude of the neon effect in Pattern $c$ is intermediate between the magnitude of the effect in Patterns $a$ and $b$. Based on these considerations, we can now formulate a twofold hypothesis: First, we hypothesize that the neon effect will not occur, notwithstanding the fact that nothing in the stimulus display will act against it, unless the neon interpretation of the pattern is the most efficient interpretation that can be made of the pattern. Second, ceteris paribus, the neon interpretation will be more prominent, and the neon effect will be stronger, to the extent that alternative interpretations of the color changes in the pattern are less efficient than the neon interpretation.

As stated above, it will be clear that, if we want to subject these hypotheses to firm experimental tests, we first of all need an objective measure for the efficiency of the different interpretations, which, presumably, affect the occurrence and the strength of the neon effect. The next section will be devoted to the principles of Leeuwenberg's coding language (Leeuwenberg, 1969, 1971), which will be used to quantify the efficiency of the pattern interpretations mentioned.

\section{THE LEEUWENBERG CODING LANGUAGE}

Although the coding system has already been described elsewhere (Leeuwenberg, 1969, 1971), it may be helfpul to present its principles again. For a formal, mathematical proof of the coding system's internal consistency and economy, the reader is referred to Buffart (Note 2).

The Leeuwenberg coding language presents both a theory on the units handled by the human pattern interpreter and a decision criterion as to the question of which one of alternative interpretations will be ultimately chosen by the interpreter. In addition, in order to represent a pattern interpretation in a pattern code, the coding language disposes of a number of notational devices. The system departs from a general and basic axiom, which says that the human pattern interpreter is first of all an efficient system. This implies that the units handled by the system are efficiently chosen. Therefore, the units proposed by the coding language refer to independent and irreducible pattern aspects. That is to say, only those pattern aspects that are not predictable from other parts of the pattern represent information that is taken into account by the pattern interpreter. In other words, only those aspects are considered relevant which represent change. In this introduction to the coding language's principles, we will use the terms "change" and "information" interchangeably. It should be noted that both terms are equivalent and that their content is defined by the specific, change-representing, coding rules to be discussed hereafter.

Another consequence of the efficiency principle concerns the decision criterion mentioned above. In accordance with that efficiency principle, the human pattern interpreter will try to interpret patterns such that the resulting interpretation is a maximally efficient one, i.e., an interpretation which contains a minimal number of change-representing information units. From the choice of maximally efficient units, it does not follow that the most efficient interpretation is arrived at automatically. If a pattern is conceived as a sequence of elements, as is done by the coding language, various sequences of elements can be proposed, which all represent the same pattern. Each sequence has its own most efficient interpretation, and from all possible interpretations of a pattern that can be made in such a way, the most efficient one is supposedly chosen by the interpreter. Notational devices will be discussed in some examples below.

\section{Reduction Principles}

If only change is treated as relevant information by the human interpreter, it follows that repetition of identical elements is not informative, because nothing changes until the sequence of identical elements comes to an end, only thereby providing information. So, almost by definition, an efficient interpretative system will look for identical elements and only register the end of such a sequence. Therefore, the first and most basic reduction principle of the Leeuwenberg coding language is repetition. 
The second reduction principle differs in only one respect from the first, in that it is concerned with identical relationships between elements instead of identical elements. If, e.g., in a sequence of different pattern elements the difference between the members of any pair of successive elements is the same, only the transition from the first element to the second element represents a real change. Because any transition after the first one is identical to the first one, it can be reduced to it by means of the first reduction rule. The second reduction rule is called integration, because identical differences are interpreted in a cumulative, integrative fashion.

The relations between groups of elements may also to some extent imply identity. A special case is the occurrence of reversal in a sequence. Reversal refers to symmetrical sequences, which are characterized by the fact that the second half repeats the elements of the first half in reversed order. The only change provided by the second half is this reversal of order of occurrence of the elements. The third reduction principle is called reversal.

According to the coding model, a pattern is first written as a sequence of pattern elements. This sequence comprises all individual pattern elements and is called the raw or primitive code of the pattern. Next, the interpreter supposedly tries to reduce this raw code as far as possible by applying code rules based on the reduction principles described above. The reduction process continues, until a final code is arrived at, one which contains only irreducible elements. The amount of structural information of the interpretation represented by the final code is defined as the number of these irreducible elements, all representing independent changes.

\section{Coding Rules and Their Notation}

Different types of repetition can be represented by means of different coding rules. Because, in this article, the coding language is applied to a rather limited set of patterns, only those coding rules will be described which are necessary to represent the information in those patterns. To differentiate between the different coding rules, different types of brackets will be used. If a more formal notational system were used, one type of brackets would be sufficient. For purposes of illustration, the notational system to be presented here is more suitable, however.

Examples of primitive codes are given in the leftmost column of Table 1 . We will not yet specify the modality or kind of patterns represented by these codes. Next to the primitive codes, one can find the reduced or final codes, which describe the primitive codes in terms of the different types of coding rules to be illustrated. The amount of structural information of each final code is given in the rightmost column.

The first primitive sequence in Table 1 shows an infinite series of elements, $\alpha$. Its final code contains one unit of information, because only the first element $\alpha$ in the series is unpredictable; all other ones are identical to the first one. Infinite repetition or continuation is indicated by brackets $\leftrightarrow \gg$ enclosing the continuing unit. As will be illustrated by some examples hereafter, the $\langle\alpha\rangle$ series can be ended by some event external to the series, or it can end itself automatically by returning to its starting point. In both cases, the end of the series needs no specification in the final code of the series itself.

The second sequence is identical to the first one, except for the fact that it is not infinite. So the end of the sequence provides extra information.

In the third example, a change occurs after the sequence of elements $\alpha$, because an element different from the preceding ones appears: $\beta$. However, because the length of the subsequence of elements $\beta$ is identical to that of elements $\alpha$, the end of the subsequence of elements $\beta$ does not provide an independent change.

In the fourth example, the lengths of the two subsequences do differ. Therefore, in that case, extra information is provided by the different numbers of $\alpha$ and $\beta$ elements. The sequence, however, can also be interpreted as made up of two subsequences of equal length plus an additional, independent element $\beta$. The final codes of both interpretations contain 4 units of information.

In the fifth example, the unit or chunk that is

Table 1

Examples of Coding Rules Used to Reduce Primitive Codes to Final Codes

\begin{tabular}{|c|c|c|c|}
\hline Primitive Code & & Final Code & $\begin{array}{c}\text { Structural } \\
\text { Information }\end{array}$ \\
\hline 1. $\quad \alpha \alpha \alpha a \ldots \ldots$ & & $\ll q\rangle$ & 1 \\
\hline 2. $\quad \alpha \alpha \alpha \alpha$ & & $4 \cdot(\alpha)$ & 2 \\
\hline 3. $\alpha \alpha \alpha \alpha \beta \beta \beta \beta$ & & $4 \cdot(\alpha \beta)$ & 3 \\
\hline \multirow[t]{2}{*}{$\alpha \alpha \alpha \beta \beta \beta \beta$} & (a) & $(34) \cdot(\alpha \beta)$ & 4 \\
\hline & (b) & $3 \cdot(\alpha \beta) \beta$ & 4 \\
\hline 5. $\alpha \beta \alpha \beta \alpha \beta$ & & $3 \cdot\{\alpha \beta\}$ & 3 \\
\hline 6. $\alpha \beta \alpha \gamma \alpha \delta$ & & $\langle(\alpha)\rangle\langle(\beta)(\gamma)(\delta)\rangle$ & 4 \\
\hline 7. 02468 & & $\int(4 \cdot(2))$ & 3 \\
\hline 8. $\alpha \beta \gamma \gamma \beta \alpha$ & & $\mathrm{R}\{\alpha \beta \gamma\}$ & 4 \\
\hline 9. $\alpha \beta \gamma \beta \alpha$ & . & $R\{\alpha \beta(\gamma)\}$ & 4 \\
\hline \multirow[t]{2}{*}{ 10. $\alpha \beta \beta \alpha \beta \alpha \beta \alpha$} & (a) & $\alpha \beta 3 \cdot\{\beta a\}$ & 5 \\
\hline & \multicolumn{2}{|c|}{ 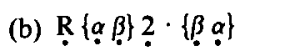 } & 6 \\
\hline
\end{tabular}

Note-A point below a code element indicates that the element in question contributes to the amount of structural information of the code. 
repeated comprises two elements. A chunk is indicated by brackets \{\} enclosing the elements which function as a group.

In the sixth example, every element of the subsequence $\beta \gamma \delta$ is preceded by an element $\alpha$. The occurrence of $\alpha$ is, therefore, informative only once, viz, the first time that it occurs. The end of this sequence is implied by the number of elements in the subsequence $\beta \gamma \delta$ and provides no information by itself.

Different types of brackets have been used to indicate different possible repetition relations between the elements of a sequence. Because it has been our argument that information is provided by the occurrence of the end of a repetition per se, and not by the kind of repetition, these brackets do not represent information by themselves. In other words, the brackets are not conceived of as independent from the other elements of the final code to which they belong.

In the seventh example in Table 1 , the difference between the elements of any pair of successive elements amounts to 2 . The sequence can be analyzed in terms of these differences between successive elements instead of in terms of the absolute values represented by the elements. The final code of this example, $\int(4 \times(2))$, now contains three units of information: one for the first element of the primitive series, one for the difference between the first and the second element, and one for the end of the repetition of these differences.

The examples on reversal (see Table 1, Nos. 8 and 9) need no further explication.

\section{Alternative Interpretations and Their Amounts of Structural Information}

By applying the coding rules mentioned above, the elements to which those rules are applied are related to each other. Therefore, an interpretation, as represented by its final code, is a specific way of relating the elements of a pattern to each other. Because in most patterns the coding rules can be applied in a number of different ways, different interpretations may result. As stated above, the most efficient, i.e., the least complex, one of these interpretations is supposedly chosen by the interpreter as the most adequate conception of the stimulus pattern. In Example 10 in Table 1, two different interpretations of the same set of primitive elements are shown. Contrary to Example 4 in the same table, in this instance one of the interpretations is more efficient than the other. The more efficient interpretation is supposedly the perceptually most preferred one. The complexity of an interpretation is defined as the number of information units in its final code. The number of information units of a final code consists of the number of symbols in the code which refer to coding rules, plus the number of symbols referring to the elements to which those rules are applied, plus the number of symbols referring to isolated, irreducible elements (an example of such an irreducible element is the last element in the final code of interpretation $4 b$ in Table 1).

\section{Application of the Coding Language to Line Pattern Interpretations}

Before going into the details of some illustrative examples, a few general remarks about the application of the coding language to line pattern interpretations have to be made. First, line patterns can be represented by sequences of line elements and angles. Primitive codes of line patterns are considered as consisting of such sequences. In the examples to be given below, angles will be represented by the first 10 letters of the Greek alphabet $(\alpha, \ldots, x)$, line elements, and numbers by the second 10 letters of the Greek alphabet $(\lambda, \ldots, v)$. The remaining letters $(\phi, \ldots, \omega)$ will be used for additional specifications, which sometimes have to be appended to line elements. Second, several aspects of line patterns will not be explicitly coded, and others, though coded, will not be considered to contribute to the amount of structural information of the interpretation coded. In the latter case, the elements in question are represented by letters from the Roman alphabet $(a, \ldots, k$ for angles; $1, \ldots, \mathbf{u}$ for line elements and numbers; $\mathrm{v}, \ldots, \mathrm{z}$ for additional specifications). If Roman letters are used in a code, those letters are to be conceived of as representing free parameters. That is to say, whichever value is substituted for them, the interpretation will remain the same.

Among the aspects of line patterns not embodied in the codes of those patterns are the spatial position of the pattern, its size or scale, the width and color of line elements, and the color of the background on which the pattern has been drawn. The latter aspects are only uninformative as long as they do not change within one and the same pattern. A complete description of a pattern interpretation would certainly have to embody all those aspects. Nevertheless, they are omitted because they are considered immaterial to the internal structure of a pattern with which interpretations are supposedly concerned in the first place. In other words, each pattern interpretation implies that there is a pattern of a certain size, drawn with some kind of ink, in one or the other orientation, somewhere on a piece of paper of any particular specification. All those aspects remain invariant within one and the same pattern, that is to say, they cannot differentiate between various interpretations of that pattern. Therefore, they are taken for granted.

In interpreting line patterns, the human perceiver supposedly tries to reduce the primitive codes, made up of angles and line elements, as far as possible by 
means of the coding rules given above. Figure 2 presents examples of line patterns, for each one of which we will discuss how its final code is arrived at. For all patterns from Figure 2, codes are given in Table 2.

Coding rule 1 (Table 1) can be applied to the first primitive code in Table 2 . The end of the series of line elements $(n)$ and angles $(\alpha)$ is specified by implication, because the series automatically returns to its starting point. Therefore, no explicit specification of the end of the series is needed in this code.

The second example in Figure 2 shows four squares arranged in a square. Each square may be interpreted in the same way as was done in the preceding example. This would result in four times the code of a square and an amount of information of four times the amount of an individual square (see Table 2, 2a). However, a different interpretation of the pattern is
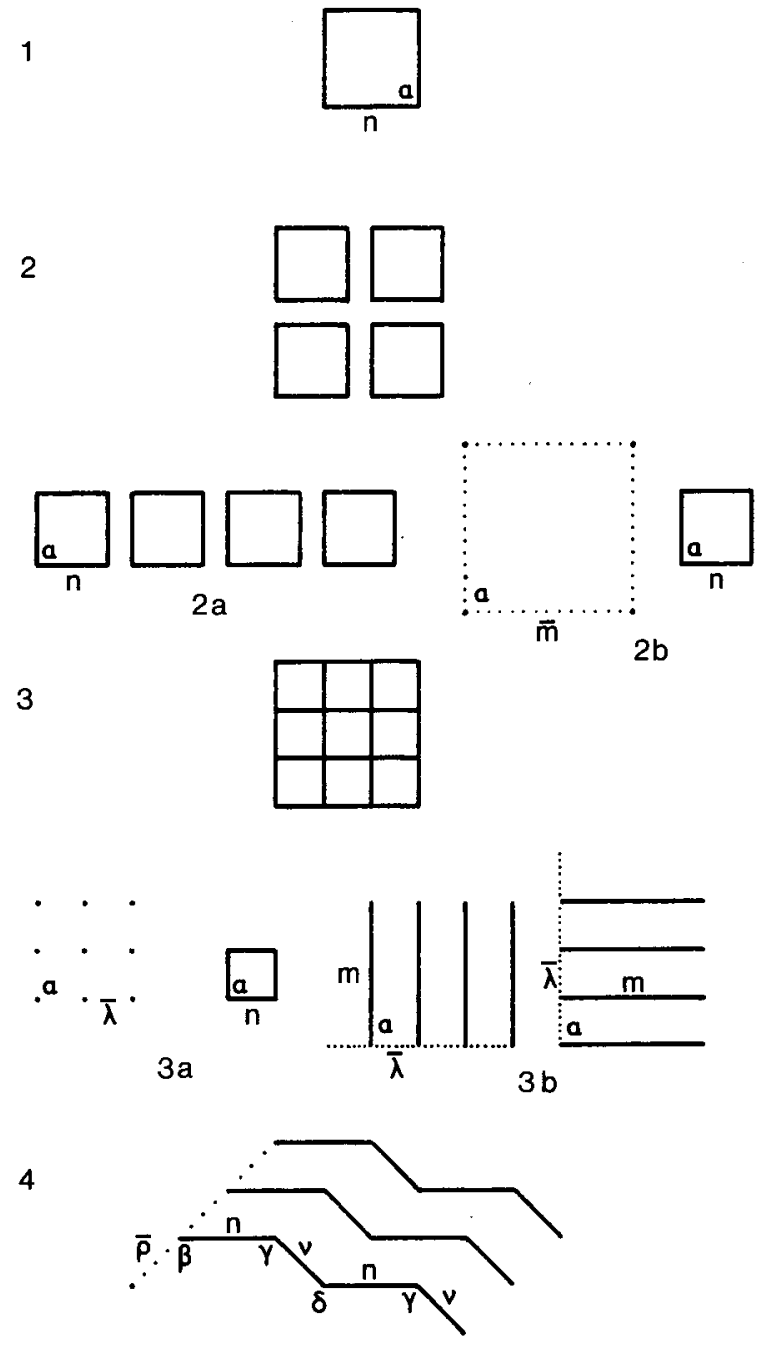

Figure 2. Examples of line patterns, the interpretations of which can be represented in a pattern code by means of Leeuwenberg's (1971) coding language (see Table 2). possible (see Table 2, 2b), one which takes into account the identity of the four squares. These squares can be conceived of as related by an invisible superstructure that specifies the positions of the squares relative to each other. The notion that a pattern is made invisible is indicated in the code by a horizontal bar above the symbols for a line element. The sequence comprising all elements of Pattern 2 consists of four distances arranged in a square, with the elements of an actual square linked to each corner of the square of distances. Brackets of the type [ ] indicate the point in the superstructure, i.e., the conceptual square of distances, to which the substructure, i.e., the actually drawn square, is attached. Neither the length of the sides of the conceptual square nor that of the actually drawn squares is informative, because both are assumed to provide only scale information. Therefore, the final code of this interpretation contains only two information units, which makes it the most efficient of the two interpretations.

The third pattern in Figure 2 shows a line pattern that can be interpreted as consisting of little squares (see 3a). However, it may also be interpreted as two sets of parallel lines (see $3 \mathrm{~b}$ ). In the first interpretation (3a), an approach is followed very much like the one in the preceding example $(2 b)$, where four little squares were attached to a hierarchically superordinated square. In the present example, the superstructure is somewhat more complicated. It consists of a regular grid of points, which is coded as a row of points, from each one of which a new row of points departs in a direction perpendicular to that of the first row. Next, a square is attached to each point of the grid. As in the preceding example (2b), the first size-specifying element of the codes of both the superstructure and the substructure is taken to be informationless. As we have already seen in the preceding example (2a), patterns can also be interpreted as consisting of independent parts. Such an interpretation is represented by code $3 b$ in Table 2 . The vertical and horizontal lines of the lattice are coded separately. Each code represents a row of distances connected by zero changes of direction to each one of which a line element of a certain length is attached in a direction perpendicular to that of the row of distances. As can be seen from Table 2, the interpretation of Pattern 3 as groups of parallel lines is the most efficient one. Notice that of each code of a (sub)pattern apprehended as an independent entity, the first scalespecifying code element at both the superstructure and the substructure level are taken to be informationless.

In our fourth example, we introduce a last important point, viz., the occurrence of line elements of different lengths. As long as all line elements in a 
Table 2

Examples of Primitive Codes and Reduced Codes of Unicolored Line Patterns Illustrated in Figure 2

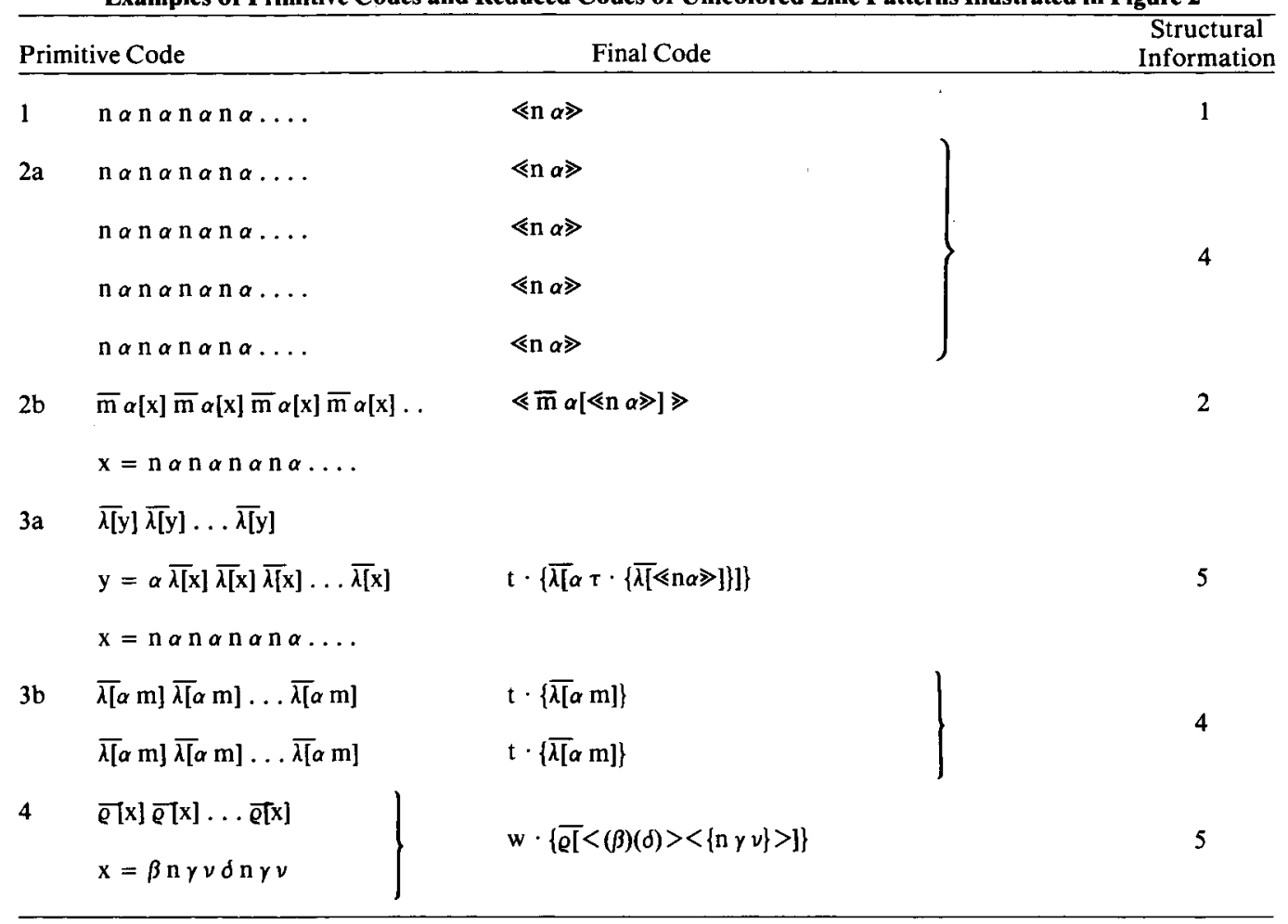

pattern are of the same length, the length of line elements cannot give rise to the detection of any change. However, if line elements of different lengths occur in a pattern, any length that differs from that of the first line element represents a change. Therefore, one unit of information is counted for the change of line element length that occurs in Pattern 4 in Figure 2.

\section{Application of the Coding Language to the Interpretation of Color Differences Occurring in Line Patterns}

As stated above, the color of the line element of a pattern does not contribute to the amount of information of a pattern interpretation until a color change occurs in the pattern. Color changes may occur between line elements as well as between parts of the background on which those line elements have been drawn. Line elements or parts of line elements with a different color can be interpreted as elements which are qualitatively different from the other elements in the pattern. According to such an interpretation, the sequence of all individual pattern elements consists of three different types of element, viz., angles, black line elements, and colored (indicated by $\chi$ or $x$ ) line elements, provided that only one deviating color is used. The amount of information in such sequences is defined, as above, as the minimum number of independent units to which the sequence of individual pattern elements can be reduced. Examples of patterns showing color differences may be found in Figure 3. Codes corresponding to these examples are given in Table 3.

The first pattern in Figure 3 consists of a sequence of angles, alternately followed by black and colored line elements. The change of direction and the change of color which occur in going from one line element to the next are the only two relevant changes in the pattern that remain after reduction of the sequence of primitive elements by applying Coding Rule 6 (see Table 1).

Another way of interpreting color differences in a line pattern is by assuming that the color is a property of an independent, colored pattern added to the uncolored or black one. An example is provided by Pattern 2 in Figure 3. Because this pattern is most efficiently interpreted as two independent subpatterns and because no color differences occur within either one of these independent subpatterns, there is no effect of the color difference in the pattern on the complexity of the interpretation. Pattern 3 in Figure 3 can be interpreted in the same way, namely, as a lattice of black lines plus an independent figure consisting of colored line elements (see 3a).

If color differences occur between the lines of a pattern as well as between the backgrounds on which these lines lie, both color differences can be interpreted as resulting from one and the same 
1

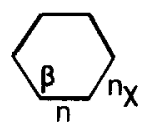

3

$3 a$

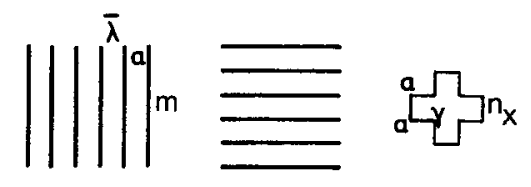

$3 b$
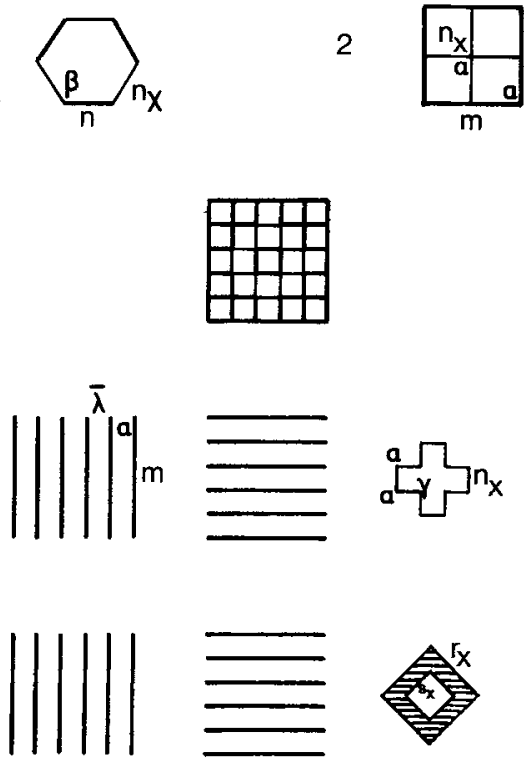

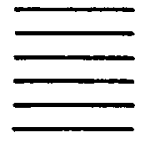

$3 c$
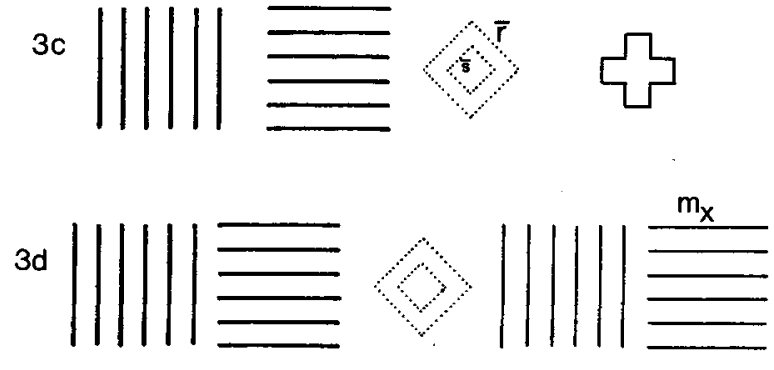

4

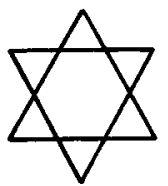

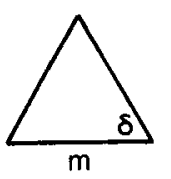

$4 a$
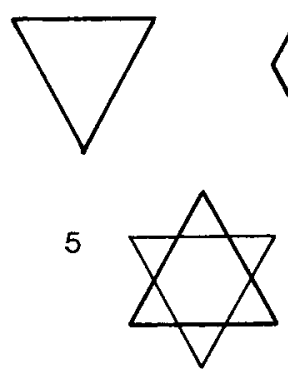
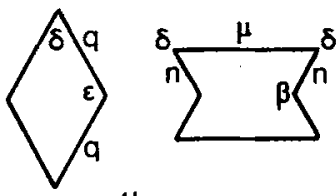

$4 b$
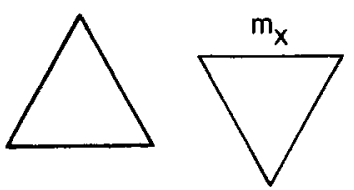

$5 a$

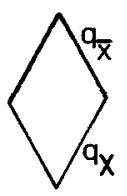

$5 b$

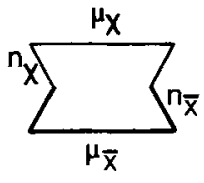

6

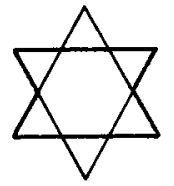

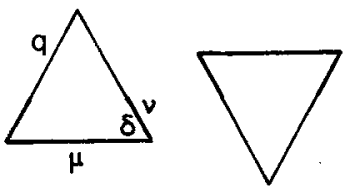

$6 a$

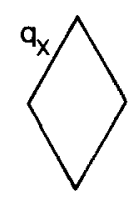

$6 b$

Figure 3. Examples of patterns with differently colored line elements. Interpretations of these patterns can be represented in a pattern code using Leeuwenberg's (1971) coding language (see Table 3).

source, such as, e.g., a colored light. See, for an example of such a (neon) interpretation, Example 3b in Figure 3 and Table 3 . The color changes that take place in the pattern can be represented by the code of a square colored area; this code can be conceived of as representing, e.g., a square hole in a screen, through which colored light is projected onto the lattice. (The inner and outer borders of the square are coded independently from each other.)

The ingredients of the neon interpretation, that is, the black lines, the colored lines, the background behind the black lines, and the differently colored background behind the colored lines, can also be interpreted in a different way. One then assumes that, instead of one unitary lattice which receives its color from an outside source, two independent, neighboring lattices are present, each drawn in a different color and on a differently colored background. The codes of two such interpretations are given in Table 3
(Examples 3c and 3d) and visualized in Figure 3 (Examples $3 c$ and 3d). A lattice of horizontal and vertical black lines, from which a square part is omitted, is represented by code 3c. The observer looks, as it were, through this hole onto a differently colored background, on which a colored, steplike structure has been drawn. Code $3 d$ represents a slightly different interpretation, in that, now, through the aperture in the black lattice, a part of another lattice, this time a colored one, is supposed to be seen.

Some explanation may be needed with regard to the contribution of color changes to the amount of information in the above interpretations. In Interpretation $3 \mathrm{~b}$, which is a neon interpretation, both the difference in line color and the difference in background color are assumed to be the result of the projection of a colored light onto the lattice and its background. Because lattice and light source are interpreted as independent parts of the total pattern, 
Table 3

Examples of Primitive Codes and Reduced Codes of Multicolored Line Patterns Illustrated in Figure 3

\begin{tabular}{|c|c|c|c|c|}
\hline & \multicolumn{2}{|c|}{ Primitive Code } & Final Code & $\begin{array}{c}\text { Structural } \\
\text { Information }\end{array}$ \\
\hline 1 & \multicolumn{2}{|c|}{$n \beta n_{\chi} \beta n \beta n_{\chi} \beta n \beta n_{\chi} \ldots$} & $\ll<(n)\left(n_{\chi}\right)><(\beta)>$ & 2 \\
\hline \multirow[t]{2}{*}{2} & \multicolumn{2}{|c|}{$\mathrm{m} \alpha \mathrm{m} \alpha \mathrm{m} \alpha \mathrm{m} \alpha \ldots$} & $\ll \mathrm{m} \alpha \gg$ & \\
\hline & \multicolumn{2}{|c|}{$\alpha n_{x} \alpha n_{x} \alpha n_{x} \alpha n_{x} \ldots$} & $\ll a\left[n_{x}\right] \gg$ & 2 \\
\hline \multirow[t]{3}{*}{$3 a$} & \multirow[t]{2}{*}{ lattice: } & $\overline{\lambda[} \alpha \mathrm{m}] \overline{\lambda[} \alpha \mathrm{m}] \ldots \overline{\lambda[} \alpha \mathrm{m}]$ & $\mathrm{t} \cdot\{\overline{\lambda[}[\alpha \mathrm{m}]\}$ & \\
\hline & & $\bar{\lambda}[\alpha \mathrm{m}] \bar{\lambda}[\alpha \mathrm{m}] \ldots \bar{\lambda}[\alpha \mathrm{m}]$ & $t \cdot\{\overline{\lambda[} \alpha \mathrm{m}]\}$ & 7 \\
\hline & cross: & $\mathrm{n}_{\mathrm{x}} \alpha \mathrm{n}_{\mathrm{x}} \alpha \mathrm{n}_{\mathrm{x}} \gamma \mathrm{n}_{\mathrm{x}} \alpha \mathrm{n}_{\mathrm{x}} \alpha \mathrm{n}_{\mathrm{x}} \gamma \ldots$ & $\ll(\alpha)(\alpha)(\gamma)>\left\langle\left(\mathrm{n}_{X}\right)\right\rangle>$ & \\
\hline \multirow[t]{3}{*}{$3 b$} & lattice: & see $3 \mathbf{a}$ & see 3a & \\
\hline & \multirow[t]{2}{*}{ square: } & $r_{x} \alpha r_{x} \alpha r_{x} \alpha r_{x} \alpha \ldots$ & $\ll \mathrm{r}_{\mathrm{x}} \alpha \gg$ & 6 \\
\hline & & $\mathrm{s}_{\mathrm{x}} \alpha \mathrm{s}_{\mathrm{x}} \alpha \mathrm{s}_{\mathrm{x}} \alpha \mathrm{s}_{\mathrm{x}} \alpha \ldots$ & $\ll s_{x} a \gg$ & \\
\hline \multirow[t]{4}{*}{$3 c$} & lattice: & see $3 \mathbf{a}$ & see $3 a$ & \\
\hline & \multirow[t]{2}{*}{ hole: } & 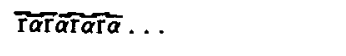 & $\langle\bar{r} \bar{\alpha}\rangle$ & 9 \\
\hline & & $\overline{S \widetilde{\alpha} \operatorname{s} \tilde{\alpha} \tilde{\alpha} \bar{S} \alpha} \ldots$ & $\langle\bar{s} \bar{\alpha} \gg$ & \\
\hline & cross: & see $3 a$ & see $3 a$ & \\
\hline \multirow[t]{4}{*}{$3 d$} & lattice: & see $3 a$ & see $3 a$ & \\
\hline & hole: & $\operatorname{see} 3 c$ & see $3 c$ & \\
\hline & \multirow{2}{*}{$\begin{array}{l}\text { colored } \\
\text { lattice: }\end{array}$} & $\left.\left.\bar{\lambda}\left[\alpha \mathrm{m}_{\mathrm{x}}\right] \overline{\lambda[} \alpha \mathrm{m}_{\mathrm{x}}\right] \ldots \overline{\lambda[} \alpha \mathrm{m}_{\mathrm{x}}\right]$ & $\left.\mathfrak{I} \cdot\left\{\overline{\lambda[} \alpha \mathrm{m}_{\mathrm{x}}\right]\right\}$ & 10 \\
\hline & & $\left.\bar{\lambda}\left[\alpha m_{x}\right] \bar{\lambda}\left[\alpha m_{x}\right] \ldots \overline{\lambda[} \alpha m_{x}\right]$ & $\left.\mathrm{t} \cdot\left\{\overline{\lambda[} \alpha \mathrm{m}_{\mathrm{x}}\right]\right\}$ & \\
\hline \multirow[t]{2}{*}{$4 a$} & \multirow{2}{*}{$\begin{array}{l}\text { triangle } \\
\text { interpre- } \\
\text { tation: }\end{array}$} & $\mathrm{m} \delta \mathrm{m} d \mathrm{~m} \delta \ldots$ & $\ll \mathrm{m} d \gg$ & \\
\hline & & $\mathrm{m} \delta \mathrm{m} \delta \mathrm{m} \delta \ldots$ & $\ll \mathrm{m} d \gg$ & 2 \\
\hline \multirow[t]{2}{*}{$4 b$} & \multirow{2}{*}{$\begin{array}{l}\text { rhomb } \\
\text { interpre- } \\
\text { tation: }\end{array}$} & $q d q \in q d q \in \ldots$ & $\ll\langle(q)\rangle\langle(\delta)(\varepsilon)\rangle\rangle$ & \\
\hline & & $\mathrm{n} \delta \mu \delta \mathrm{n} \beta \mathrm{n} \delta \mu \delta \mathrm{n} \beta \ldots$ & $\ll R\{n d(\mu)\} \beta \gg$ & 6 \\
\hline \multirow[t]{2}{*}{$5 a$} & \multirow{2}{*}{$\begin{array}{l}\text { triangle } \\
\text { interpre- } \\
\text { tation: }\end{array}$} & see $4 a$ & $\operatorname{see} 4 a$ & \\
\hline & & $m_{x} \delta m_{x} \delta m_{x} \delta \ldots$ & $\ll \mathrm{m}_{\mathrm{x}} \delta \gg$ & 2 \\
\hline \multirow[t]{2}{*}{$5 b$} & \multirow{2}{*}{$\begin{array}{l}\text { rhomb } \\
\text { interpre- } \\
\text { tation: }\end{array}$} & $\{q \delta q \varepsilon\}_{\bar{x}}\{q \delta q \varepsilon\}_{\chi} \ldots$ & $\ll<(<(q)\rangle\langle(d)(\varepsilon)>)>\langle(\bar{x})(\chi)>\gg$ & 8 \\
\hline & & $\begin{array}{l}\{n \delta \mu \delta n \beta\}_{\bar{x}}\{n \delta \mu \delta n \beta\}_{\chi} \cdots \\
\bar{x}=\text { black }\end{array}$ & $\ll<(\mathrm{R}\{\mathrm{n} \delta(\mu)\} \beta)><(\mathrm{x})(\chi)>\gg$ & 8 \\
\hline \multirow[t]{2}{*}{$6 \mathbf{a}$} & \multirow{2}{*}{$\begin{array}{l}\text { triangle } \\
\text { interpre- } \\
\text { tation: }\end{array}$} & $\mathrm{q}_{\mathrm{x}} v \delta \mu \delta \nu \mathrm{q}_{\mathrm{x}}$ & $\mathbf{R}\left\{\mathbf{q}_{x} \nu \delta(\mu)\right\}$ & \\
\hline & & $\mathrm{q}_{x} \nu \delta \mu \delta \nu \mathrm{q}_{x}$ & $\mathbf{R}\left\{\mathrm{q}_{\chi} v \delta(\mu)\right\}$ & 10 \\
\hline \multirow[t]{2}{*}{$6 b$} & \multirow{2}{*}{$\begin{array}{l}\text { rhomb } \\
\text { interpre- } \\
\text { tation: }\end{array}$} & $q_{x} \delta q_{x} \varepsilon q_{x} \delta q_{x} \varepsilon \ldots$ & $\ll\left\langle\left(q_{x}\right)\right\rangle\langle(\delta)(\varepsilon)\rangle \gg$ & \\
\hline & & $\operatorname{see} 4 b$ & $\operatorname{see} 4 b$ & 6 \\
\hline
\end{tabular}


all color changes are between-pattern and not within-pattern changes. Therefore, they do not contribute to the amount of information of the interpretation, contrary, e.g., to a preceding example (see Pattern 1 in Figure 3), in which the change of line color was interpreted as a change within an independent pattern. In Interpretation $3 c$, as well as in $3 \mathrm{~d}$, the color change occurring in the background is interpreted as the result of the presence of two differently colored backgrounds, one lying behind and partly covered by the other. Again, because of the independence of the two backgrounds, the color difference between the two does not contribute to the information of the interpretation. The same holds true for the color difference between the lines of the two groups of lines in the pattern.

The color differences discussed above are, as such, most certainly noticed by every observer or pattern interpreter, and they most certainly have an influence on the interpretation of the patterns in which they occur. However, they do so by increasing the information load of alternative interpretations, according to which the differently colored elements are related to each other. We will illustrate this with two colored versions of Pattern 4 in Figure 3. A triangle is more easily detected in one colored version than it is in the uncolored version (Pattern 5). Yet the code for the interpretation, "two triangles," is identical for both patterns. It is more difficult to find a rhomb figure in Pattern 5 than in Pattern 4, because, in Pattern 5, color differences within the rhomb contribute to the complexity of the interpretation of the pattern as a rhomb figure plus another figure. In Pattern 6, the triangle can hardly be found anymore, whereas the rhomb figure is now the most easily found subpattern. This time, the color differences complicate the triangle interpretation, because the base of each triangle and parts of the other sides are differently colored. In general, if color differences increase the information load of one interpretation, they also decrease at the same time the complexity of other interpretations by increasing the difference between them.

In summary, colored line elements in an otherwise black pattern can be interpreted as discrete pattern elements in a coherent, independent pattern. Such an interpretation will often be efficient if color changes in the pattern parallel form changes (see Pattern 1 in Figure 3). Colored line elements can also be interpreted as making up an independent structure themselves (see Pattern 2 in Figure 3). A third way of interpreting color differences is by means of a colored figure projected upon or placed in front of the otherwise uncolored lattice (neon interpretation; see Interpretation $3 \mathrm{~b}$ in Figure 3 ). This interpretation will be possible only if color differences appear to be present both between line elements and between the backgrounds on which these line elements lie. In that case, also, a fourth interpretation is possible, viz., one according to which the pattern consists of independent, differently colored backgrounds, each one overlayed with its own line structure (see Interpretations $3 c$ and $3 d$ in Figure 3 ). In the next section, the role each of the four above interpretations plays in the determination of a pattern's predicted neon prom. inency will be discussed.

\section{Predicted Neon Prominency, P(n)}

Following our original hypothesis, which says that the occurrence of the neon effect is dependent on the relative efficiency of the neon interpretation, we predict that no neon effect will occur in a pattern if an alternative, nonneon interpretation is more efficient than the neon interpretation of that pattern. Examples of nonneon interpretations (Nos. 1, 2, and 3a) and an example of a neon interpretation (3b) can be found in Figure 3 and Table 3 . In addition, it is predicted that the absence of neon in a pattern will be more compelling, to the extent that the neon interpretation is relatively more complex than the most efficient nonneon interpretation. We therefore suppose that the discard of the most efficient interpretation of a pattern in favor of a less efficient one is more difficult, to the extent that the latter is relatively more complex. In accordance with this line of reasoning, it is predicted that the occurrence of neon is dubious in cases in which neon and nonneon interpretations are equally efficient. In formula:

$$
\text { If } I(n n) \leqslant I(n) \text {, then } P(n)=\frac{I(n n)-I(n)}{I(n n)} \leqslant 0,
$$

in which: $I(n n)=$ the amount of structural information of the most efficient nonneon interpretation that can be given of the pattern, $I(n)=$ the amount of structural information of the neon interpretation of the pattern, and $P(n)=$ the prominency of the neon interpretation of the pattern.

On the other hand, if the neon interpretation is the most efficient interpretation possible, it is predicted that the neon effect will indeed occur. Now, before presenting more detailed predictions, we have to bring up a problem that concerns the primitive data on which the neon interpretation is to be based. As explicated in the section on the coding language, a final code, representing a certain pattern interpretation, always departs from a set of primitive data. In case of a neon interpretation, these primitive data should at least include differently colored line elements and differently colored backgrounds. In other words, if the latter were absent, a neon interpretation would be at variance with the raw data. Now, if black and colored line elements have actually been drawn on a homogeneously colored background, 
this background can only get different colors by the occurrence of line spreading around the black and colored line elements. Therefore, if we want to add differently colored backgrounds to the primitive data of a pattern with differently colored lines that have actually been drawn on a homogeneously colored background, we need to know if the conditions for the occurrence of line spreading are met. However, nothing is definitely known about those conditions, so one can never be sure if one is coding a neon interpretation correctly. The problem raised here can only be solved by assuming that the conditions for the occurrence of line spreading parallel those for the occurrence of the neon effect. In other words, if the neon effect is dependent on organizational factors, spreading must somehow be dependent on such factors too. Otherwise, we should expect to find patterns that do not show a neon effect, notwithstanding that the conditions for the occurrence of neon, as formulated above, are met.

The assumption that the conditions for line spreading will be fulfilled if a neon interpretation is the most efficient interpretation that can be given of a pattern has an important consequence. Because, in such cases, differently colored background areas are part of the primitive data, all interpretations of the patterns in question have, in order to be adequate, to take into account those background color differences. In other words, if a neon interpretation is most efficient, interpretations such as $3 a$ in Figure 3 and Table 3 are simply no longer allowed, because they do not represent all primitive data present in the pattern. On the other hand, interpretations such as $3 \mathrm{c}$ and $3 \mathrm{~d}$ (see Figure 3 and Table 3 ) do account for spreading effects, and therefore, they can be considered adequate alternative interpretations in cases in which the neon interpretation is the most efficient interpretation possible.

Based on the above considerations, we can now predict neon prominency in more detail for cases in which a neon effect is supposed to be present:

$$
\text { If } I(n)<I(n n) \text {, then } P(n)=\frac{I(\text { as })-I(n)}{I(\text { as })}>0 \text {, }
$$

in which: $\mathrm{I}(\mathrm{as})=$ the amount of structural information of the most efficient alternative interpretation of line spreading effects in a pattern.

In summary, we propose a two-stage analysis of potentially neon-displaying patterns. First, it has to be established if a nonneon interpretation is probably so efficient that a neon interpretation of the color differences between the lines of a pattern would always be equally or more complex. In such cases, it is predicted that neon prominency will be zero or negative. Second, if no interpretation more efficient than the neon interpretation can be given of a pattern, it is predicted that neon will arise, and that the prominency of the neon effect will be a function of the efficiency of the neon interpretation and the efficiency of an interpretation which organizes the color differences between the line elements as well as the color spreading effects, which are apparently present, in an alternative way. See, for an illustration, Pattern 3 in Figure 3. The codes corresponding to the different possible interpretations of that pattern are given in Table 3. Because the nonneon interpretation (see Figure 3a) is more complex than the neon interpretation (see Figure 3b), a neon effect is predicted to occur. An estimate of its prominency is arrived at by relating the complexity of the neon interpretation to the complexity of the most efficient alternative spreading interpretation (see Figure 3c). For this pattern, we find a predicted neon prominency of .33.

It should be noticed that alternative spreading interpretations are, by definition, special cases of nonneon interpretations. The reason for the differentiation is that, in our opinion, not all nonneon interpretations, especially not those which imply the absence of spreading, can be legitimately used in the determination of neon prominency in cases in which the occurrence of spreading has to be assumed.

The experiments to be described next were designed to test the adequacy of the coding-language-based prominency measures proposed above.

\section{EXPERIMENT 1}

\section{Method}

Subjects. The subjects were 36 undergraduate psychology students, who took part in the experiment in partial fulfillment of a course requirement.

Materials. Stimuli consisted of 16 pairs of line patterns, drawn with black and colored drawing pens on white cardboard. The patterns measured, on the average, $9 \times 9 \mathrm{~cm}$. Line width was approximately $.8 \mathrm{~mm}$. Each pair was drawn on a separate piece of cardboard, one pattern at the left, the other one at the right side, with a distance of $2.5 \mathrm{~cm}$ between patterns. Within each pair, structural relationships between black and colored line elements were varied, whereas metrical properties, such as line width, line separation, number of black and colored line elements, background luminance, line color, and line luminance were held constant.

For each pattern of a pair, the amounts of structural information of its most efficient nonneon and neon interpretation were determined. If the nonneon interpretation was more efficient than the neon interpretation, or equally efficient, the (negative) prominency of the neon effect was determined by means of Formula 1 (see preceding section). If the neon interpretation was the more efficient one, the amount of structural information of the most efficient alternative spreading interpretation was determined and then the prominency of the neon effect was calculated by means of Formula 2 (see preceding section). Predicted prominency values, $P(n) a$ and $P(n) b$, for the $a$ and $b$ members of each pair may be found in Table $4 .^{3}$

Procedure. The general method was to have subjects compare the strength of the neon effect in the paired displays. Each subject was first familiarized with the neon phenomenon by some illustrative examples. The defining features of the effect were explicitly 
Table 4

Amount of Structural Information (I) of the Nonneon (nn) Interpretation, the Alternative Spreading (as) Interpretation, and the Neon (n) Interpretation, as Far as Necessary to Determine Each Pattern's Predicted Prominency [P(n)], and Response Frequencies (f) to the Patterns of Each Pair

\begin{tabular}{|c|c|c|c|c|c|c|c|c|c|c|c|}
\hline Pattern & I(nn) & I(as) & $\mathrm{I}(\mathrm{n})$ & $\mathrm{P}(\mathrm{n})$ & f & Pattern & I(nn) & I(as) & $\mathrm{I}(\mathrm{n})$ & $P(n)$ & f \\
\hline $1 \mathrm{a}$ & $9 *$ & 10 & 6 & .400 & 28 & $9 a$ & $8^{*}$ & 9 & 7 & .222 & 25 \\
\hline $1 \mathrm{~b}$ & 10 & & 14 & -.286 & 8 & $9 b$ & 7 & & 7 & .000 & 11 \\
\hline $2 a$ & $6 *$ & 6 & 4 & .333 & 29 & $10 \mathrm{a}$ & $9 *$ & 10 & 8 & .200 & 30 \\
\hline $2 b$ & 6 & & 8 & -.250 & 7 & $10 \mathrm{~b}$ & 7 & & 7 & .000 & 6 \\
\hline $3 \mathbf{a}$ & $7^{*}$ & 9 & 5 & .444 & 28 & $11 \mathrm{a}$ & $5^{*}$ & 5 & 4 & .200 & 23 \\
\hline $3 b$ & 7 & & 8 & -.125 & 8 & $11 b$ & 4 & & 4 & .000 & 13 \\
\hline $4 a$ & $10^{*}$ & 12 & 8 & .333 & 29 & $12 \mathrm{a}$ & $9^{*}$ & 10 & 7 & .300 & 28 \\
\hline $4 b$ & 10 & & 12 & -.167 & 7 & $12 \mathrm{~b}$ & $8^{*}$ & 9 & 7 & .222 & 8 \\
\hline $5 a$ & $7^{*}$ & 9 & 5 & .444 & 29 & $13 a$ & $6^{*}$ & 6 & 4 & .333 & 28 \\
\hline $5 b$ & 7 & & 7 & .000 & 7 & $13 b$ & $8^{*}$ & 7 & 5 & .286 & 8 \\
\hline $6 a$ & $7^{*}$ & 9 & 5 & .444 & 31 & $14 a$ & $8^{*}$ & 10 & 6 & .400 & 31 \\
\hline $6 \mathrm{~b}$ & 10 & & 10 & .000 & 5 & $14 b$ & $8^{*}$ & 10 & 6 & .400 & 5 \\
\hline $7 a$ & $4 *$ & 5 & 3 & .400 & 28 & $15 \mathrm{a}$ & $12^{*}$ & 9 & 5 & .444 & 22 \\
\hline $7 b$ & 5 & & 5 & .000 & 8 & $15 b$ & $9 *$ & 9 & 5 & .444 & 14 \\
\hline $8 a$ & $5^{*}$ & 6 & 4 & .333 & 27 & $16 \mathrm{a}$ & 6 & & 6 & .000 & 12 \\
\hline $8 b$ & 6 & & 6 & .000 & 9 & $16 \mathrm{~b}$ & 6 & & 6 & .000 & 24 \\
\hline
\end{tabular}

*These numbers were not used in the calculation of $P(n)$, but only in the calculation of $P(n) 4$ (see General Discussion).

mentioned to him. A neon-displaying pattern should raise the impression of a homogeneously colored lattice, partly discolored by a light beam projected upon it. The structure of the group of discolored lattice lines should be difficult to identify. Color differences should be observable between lines of the lattice and between parts of the background behind those lines. He was then asked to use those features in judging which member of each of the 16 pairs showed more neon effect. The subjects went once through the series, each subject in a different random order. The subjects responded by pointing to the pattern which, in their opinion, showed the stronger neon effect. The pattern with the theoretically stronger neon effect was half of the time drawn at the left and half of the time at the right side of each card. The cards were placed vertically in a card holder in front of the subject, who sat at a distance of $1.5 \mathrm{~m}$ from the card holder. Illumination was by normal fluorescent lamps. Presentation time was unlimited, but the subjects were urged to make their decisions as fast as possible.

\section{Results and Discussion}

Frequencies of responses to each pattern of the $\mathbf{1 6}$ pairs are given in Table 4. From this table, it can be seen that in all 13 cases in which a difference in strength of the neon effect between the members of a pair was predicted, a majority of subjects responded in accordance with those predictions. All differences in response frequencies were significant at the .01 level, except for Pair 11 ( $p<.07$ ), as tested by the binomial test. In cases where no differences were predicted (Pairs 14, 15, and 16), a majority of subjects nevertheless judged one of the patterns as displaying a stronger neon effect. In two cases (Pairs 14 and 16), differences were significant $(p<.01)$ or nearly significant $(p<.07)$, as tested by the binomial test (two-sided).

As far as these results are in agreement with the predictions, they present only a rather weak test of the proposed prominency measures. The reason is that, for a majority of pairs, the pattern with the higher prominency is at the same time the only one for which a neon effect is predicted at all. Positive results, therefore, are more in conformity with the more general hypothesis that the occurrence of neon is governed by interpretational factors, than with the more specific assertion that the magnitude of the effect is determined by those factors too. In order to investigate how accurately the magnitude of the neon effect can be predicted by means of prominency measures, a second experiment was done in which the 16 pairs were rank ordered according to the degree of difference in neon effect between the patterns of each pair. The results of that experiment will also enable us to evaluate how serious the inconsistencies in Pairs 14 and 16 are to be taken. Discussion of those pairs will, therefore, be postponed until then.

\section{EXPERIMENT 2}

Nonstructural factors, such as line width, line separation, and the number of black and colored line elements in a pattern, probably contribute to the magnitude of the neon effect, as they contribute to the Von Bezold spreading effect (Helson, 1963). Because those factors were held constant only within pairs, it is not possible to directly compare with each other all 32 patterns used in Experiment 1 on the magnitude of the neon effect displayed by them. For that reason, only within-pair comparisons were made in that experiment. If the contribution of nonstructural factors to the magnitude of the neon effect is equal for both patterns within a pair, the degree of the difference in neon effect between the members of a pair can be predicted by taking the difference of the prominency values of the members of that pair. If we do so for every pair, we can predict the order of those differences in neon effect strength over pairs without knowing the actual contribution of nonstructural factors to the effect within each pair. 


\section{Method}

Subjects. The subjects were 14 undergraduate psychology students who took part in the experiment in partial fulfillment of a course requirement. None of them had served as a subject in the preceding experiment.

Materials and Procedure. Stimuli were identical to those in Experiment 1. Prominency values for the patterns of each of the 16 pairs can be found in Table 4. By taking the difference of the prominency values of Patterns $a$ and $b$ of each pair, $P(n) a-P(n) b$, the predicted prominency difference, $P(n) d$, was determined for each pair.

Each subject was familiarized with the neon effect in the same way as was done in Experiment 1. Next, the subject was led to one end of a large table, on which all 16 pairs had been laid down in a row, in a different random order, for each subject. The subject had to go along the table once, looking carefully at the differences in neon strength between the patterns of each pair (it had been emphasized in the instructions that the subject should only be concerned with differences; small differences between patterns that both showed a rather strong neon effect should be judged equal to small differences between patterns that both showed a rather weak neon effect). Having seen all pairs once, the subject went along the table a second time in order to divide the $\mathbf{1 6}$ pairs into two groups, one with large-difference pairs, the other with small-difference pairs. This procedure was repeated with each of the two groups, until all 16 pairs had been ordered from large to small. The subjects were allowed to rearrange pairs between groups, if such would, in their opinion, result in a better ordering.

\section{Results and Discussion}

Table 5 contains the rank orderings of the 16 pairs by 14 subjects. From the table, it can be seen that there is high agreement among subjects with regard to the question of which pairs display large differences and which pairs display small differences in neon strength. If we subdivide the total set of 16 pairs, on the basis of their $P(n) d$ values, into a subset of 8 pairs with large predicted differences and a subset of 8 pairs with small predicted differences, we see that the observed dichotomy corresponds rather well with the predicted dichotomy. Of 112 pairs placed in the large difference subset, 100 were pairs with large predicted differences in neon strength $\left(\chi^{2}=69.143 ; p<.01\right)$. The only pairs which are not classified by a significant majority of subjects (i.e., at least 11 out of 14 subjects) as belonging to one or the other of our artificial subsets are Pairs 8 and 9. If there is a continuous variation in difference prominency, as our $P(n) d$ values suggest, we should indeed expect to find some patterns difficult to classify unambiguously because they lie close to the boundary between the two subsets.

Overall agreement among subjects appeared to be highly significant, as tested by Kendall's coefficient of concordance $(W=.75 ; p<.01)$. By taking the median rank number of each pair as an index of its overall observed prominency difference, the rank correlation between predicted and observed difference in neon strength between the patterns of the 16 pairs could be determined. The rank correlation coefficient found was as high as .99. Rank correlation coefficients for individual subjects ranged from .72 to .98 (see Table 5). (For all rank correlation coefficients, $\mathrm{p}<.01$.)

As far as individual subjects are concerned, the prominency measure fairly accurately predicts which differences in neon strength are judged large and which small. Summing the results of even a rather small $(n=14)$ group results in very accurate predictions for the total range of differences investigated. We therefore feel justified in concluding that structural factors, as represented by the alternative pattern interpretations in the prominency measure, determine to a large extent the magnitude of the neon illusion.

Table 5

Rank Orderings of the 16 Pairs According to the Magnitude of the Difference of Neon Effect Between the Members of Each Pair

\begin{tabular}{|c|c|c|c|c|c|c|c|c|c|c|c|c|c|c|c|c|c|}
\hline \multicolumn{18}{|c|}{ Pairs } \\
\hline \multirow[b]{3}{*}{ Subjects } & 1 & 2 & 3 & 4 & 5 & 6 & 7 & 8 & 9 & 10 & 11 & 12 & 13 & 14 & 15 & 16 & \\
\hline & \multicolumn{17}{|c|}{$P(n) d$} \\
\hline & .686 & .583 & .569 & .500 & .444 & .444 & .400 & .333 & .222 & .200 & .200 & .078 & .047 & .000 & .000 & .000 & $\mathrm{r}^{*}$ \\
\hline 1 & 1 & 2 & 4 & 3 & 6 & 5 & 7 & 8 & 9 & 11 & 10 & 13 & 14 & 16 & 12 & 15 & .98 \\
\hline 2 & 1 & 4 & 3 & 2 & 6 & 5 & 7 & 13 & 8 & 10 & 12 & 9 & 11 & 15 & 14 & 16 & .92 \\
\hline 3 & 1 & 4 & 6 & 2 & 5 & 7 & 3 & 13 & 8 & 9 & 10 & 11 & 12 & 15 & 14 & 16 & .90 \\
\hline 4 & 6 & 1 & 2 & 7 & 3 & 4 & 5 & 8 & 11 & 9 & 12 & 10 & 14 & 13 & 16 & 15 & .90 \\
\hline 5 & 1 & 7 & 3 & 2 & 5 & 4 & 6 & 12 & 8 & 11 & 15 & 9 & 10 & 14 & 16 & 13 & .86 \\
\hline 6 & 3 & 2 & 5 & 8 & 7 & 4 & 1 & 6 & 11 & 13 & 9 & 12 & 10 & 14 & 16 & 15 & .86 \\
\hline 7 & 2 & 6 & 3 & 1 & 4 & 5 & 7 & 9 & 10 & 8 & 15 & 14 & 16 & 12 & 13 & 11 & .85 \\
\hline 8 & 2 & 5 & 7 & 1 & 4 & 3 & 8 & 10 & 9 & 6 & 15 & 11 & 13 & 14 & 16 & 12 & .85 \\
\hline 9 & 2 & 3 & 5 & 1 & 6 & 7 & 10 & 9 & 12 & 4 & 8 & 11 & 16 & 15 & 13 & 14 & .85 \\
\hline 10 & 2 & 1 & 3 & 6 & 4 & 5 & 7 & 8 & 15 & 9 & 16 & 10 & 13 & 11 & 14 & 12 & .84 \\
\hline 11 & 5 & 2 & 1 & 9 & 3 & 7 & 6 & 4 & 8 & 11 & 15 & 12 & 13 & 10 & 16 & 14 & .82 \\
\hline 12 & 5 & 2 & 1 & 6 & 3 & 8 & 4 & 15 & 9 & 12 & 7 & 11 & 13 & 10 & 16 & 14 & .80 \\
\hline 13 & 1 & 4 & 3 & 2 & 8 & 6 & 10 & 11 & 7 & 14 & 13 & 5 & 12 & 15 & 9 & 16 & .79 \\
\hline 14 & 9 & 2 & 1 & 3 & 6 & 7 & 8 & 4 & 12 & 16 & 5 & 13 & 11 & 15 & 10 & 14 & .72 \\
\hline \multicolumn{18}{|l|}{ Median } \\
\hline Rank & 2 & 2.5 & 3 & 2.5 & 5 & 5 & 7 & 9 & 9 & 10.5 & 12 & 11 & 13 & 14 & 14 & 14 & .99 \\
\hline
\end{tabular}

*Correlation between predicted and observed rank orders. 
Returning to the inconsistencies found in Experiment 1 , in which the direction rather than the degree of the difference in neon strength between the patterns of each pair was investigated, we see that the pairs with inconsistent results have all been placed at the small-difference end of the continuum now, which is in accordance with the predicted zero differences of these pairs. We therefore believe that the inconsistent findings in those pairs in Experiment 1 need no further discussion.

\section{GENERAL DISCUSSION}

The results of the preceding experiments support the position outlined in the introduction: Occurrence and strength of the neon effect depend to a large extent on interpretational factors. Quantification of those factors by means of Leeuwenberg's coding language appears to be a fruitful approach for several reasons. In the first place, theorizing about effects of organizational or interpretational factors is raised above the level of noncommittal propositions, because, for the first time, falsification of such propositions has become possible. Secondly, we can, e.g., by systematically investigating the relative efficiency of various possible prominency measures, further our insights into the qualitative aspects of the processing of visual information.

Table 6 provides an illustration of the latter point. The table contains coefficients for the rank correlation of each of four prominency measures with the overall prominency ratings obtained in Experiment 2. Correlation coefficients have been determined for the full range of 16 pairs, as well as for the 8 pairs judged to show large differences and the 8 pairs judged to show small differences in neon strength. From the table it can be seen that the highest correlation coefficients are obtained if the procedure of prominency calculation proposed in this article is followed [see Table 6, $P(n) 1]$. If one takes, as an alternative prominency measure, the absolute difference between the efficiency of the neon and the alternative interpretation of a pattern, lower correlations are obtained [see Table 6, P(n)2], especially for the subset of large difference pairs. On the basis of such a result, the hypothesis might be raised that the visual system measures efficiency in relative rather than in absolute terms.

Lower correlations are also obtained, if we do not let prominency values become less than zero. Such a rule could be based upon the argument that in the absence of neon it is of no use to determine any prominency at all. From Table 4 it can be seen that negative prominency values are obtained for the $b$ patterns of each of the first four pairs. If those negative values are replaced by zeros, the $P(n) d$ values change substantially and the high correlation between
Table 6

Correlation Between Four Different Prominency Measures and Observed Prominency Ratings

\begin{tabular}{lcccc}
\hline & \multicolumn{4}{c}{ P(n) } \\
\cline { 2 - 5 } & 1 & \multicolumn{1}{c}{2} & \multicolumn{1}{c}{3} & 4 \\
\hline Total Range of 16 Pairs & .99 & .98 & .86 & .97 \\
Subset of Large Difference Pairs & .96 & .89 & -.13 & .96 \\
Subset of Small Difference Pairs & .96 & .95 & .96 & .85 \\
\hline
\end{tabular}

predicted and observed prominency difference totally disappears for the subset of large difference pairs [see Table 6, P(n)31. By this fact, the somewhat laborious procedure of calculating different prominency measures, depending upon the difference in efficiency of the neon and the nonneon interpretation of a pattern, is justified. Negative prominency may be interpreted as the extra trouble which the visual system would have to take in bringing about a neon interpretation if a more efficient interpretation is available.

Inspection of the four patterns with negative neon prominency reveals that in all four cases the colored line elements are most efficiently interpreted as belonging to independent colored figures. ${ }^{3}$ At the same time, it can be seen that there is neither neon nor any color spreading at all in those patterns. The hypothesis might be raised that spreading is a characteristic of line elements that function as a background, whereas the potential spreading around line elements that function as a figure probably is inhibited. The fact that the neon effect disappears under fixation fits in with this kind of reasoning. The observation made here may be used as a point of departure for the investigation of the general conditions for the occurrence of spreading which should encompass the conditions for the occurrence of the neon effect.

As a third alternative, we have examined a prominency measure which compares the efficiency of the neon interpretation with that of the shortest of two other interpretations, viz., the nonneon interpretation and the alternative spreading interpretation. Amounts of structural information of the interpretations used to calculate $P(n) 4$ can be found in Table 4 . As can be seen from Table $6[P(n) 4]$, this prominency measure results in lower correlations too, especially for the subset of small difference pairs.

This finding suggests that, in cases in which the neon interpretation was the most efficient interpretation possible, we rightly based the alternative interpretation on a set of primitive data which included spreading, i.e., color differences between parts of the background on which the pattern had been drawn. So, if the neon interpretation is the most efficient one possible, the most successful neon prominency measure seems to be one which, in essence, compares two alternative interpretations of 
spreading, viz., the neon interpretation and the alternative spreading interpretation. According to the latter, color differences, resulting from spreading, are interpreted as properties of the background on which the colored lines lie. This situation is reminiscent of the Von Bezold spreading phenomenon (Von Bezold, 1874). According to the neon interpretation, color differences are the result of illumination by an outside source. If the neon interpretation is the most efficient one, spreading apparently gets its specific neon quality. As the alternative spreading interpretation becomes more efficient, the specific neon quality is replaced by the impression of differently colored background areas, each one overlayed with its own line structure (Von Bezold effect).

Our prominency measure, which contrasts two interpretations of spreading, is probably so successful because a third qualitatively different interpretation of spreading cannot easily be given. Therefore, a prominency measure based on a two-alternative contrast is probably only relevant to situations in which the choice of the final pattern interpretation is a choice from two alternatives.

\section{REFERENCE NOTES}

1. Tuijl, H. F. J. M. van, \& Weert, Ch. M. M. de. Sensory conditions for the occurrence of the neon spreading illusion. Manuscript submitted for publication, 1978.

2. Buffart, H. F. J. M. A coding language for patterns. Report 73FU07, University of Nijmegen, The Netherlands, 1973.

\section{REFERENCES}

Beck, J. Contrast and assimilation in lightness judgments. Perception \& Psychophysics, 1966, 1, 342-344.

Helson, H. Studies of anomalous contrast and assimilation. Journal of the Optical Society of America, 1963, 53, 179-184.

KoffKa, K. Principles of Gestalt psychology. London: Routledge \& Kegan Paul, 1935.

LEEUWENBERG, E. L. J. Quantitative specification of information in sequential patterns. Psychological Review, 1969, 76, 216-220.

LEEUWENBERG, E. L. J. A perceptual coding language for visual and auditory patterns. American Journal of Psychology, 1971, 84, 307-349.

TuIJ, H. F. J. M. vav. A new visual illusion: Neonlike color spreading and complementary color induction between subjective contours. Acta Psychologica, 1975, 39, 441-445.

Von Bezold, W. Die Farbenlehre im Hinblick auf Kunst und Kunstgewerbe. Braunschweig: Westermann, 1874.

\section{NOTES}

1. All illustrations in this article were prepared in black and white, with heavy and thin lines instead of black and colored lines of equal width. As demonstrated before (Van Tuijl, 1975), both neon-like color spreading and neon-like brightness spreading can occur. Although using black and white demonstrations, we will always refer in the text to the color phenomenon, because all of the experimentation was done with color displays.

The reader should realize that by using lines of unequal width to induce brightness variations, the neon phenomenon loses quality as compared with illustrations that are prepared with lines of equal width. As will be understood from the remainder of this article, the reason is that lines of different width interfere with the interpretation of patterns as unitary lattices of lines. This latter interpretation is a prerequisite for the neon effect.

2. The most well-known spreading effect is certainly Von Bezold's color spreading effect, discovered more than a century ago by Von Bezold (1874). In the Von Bezold pattern, a homogeneously colored background is overlayed with two line structures drawn. adjacent to each other, one in black, the other one in white. The color of the background behind the black lines is perceived as darker and that behind the white lines as lighter than a matching stimulus, which is physically identical to the color behind both line structures. The difference in impression raised by Von Bezold's and Van Tuijl's patterns lies, first, in the fact that the former is not perceived as one unitary line structure. On the contrary, black and white lines make up two distinct groups of lines, each one with a clearly recognizable structure. In addition, the illusory color difference between the two background areas behind the black and white lines in the Von Bezold pattern is not perceived as the result of illumination by an outside source. Rather, one has a genuine impression of two, differently colored, background areas.

Apart from the necessary conditions for the occurrence of a neon effect, which will be discussed in the present article, it has been possible to formulate some preconditions for the neon effect in terms of luminance relations between the two groups of line elements in the pattern and the background on which they have been drawn (Van Tuijl \& de Weert, Note 1).

3. An appendix with all the experimental patterns, representations of their alternative interpretations, and the codes belonging to those interpretations is available from the authors upon request.

(Received for publication July 21, 1978; accepted November 20,1978 .) 